\title{
Ordering in Two-Dimensional Lennard-Jones Clusters
}

\author{
Barnana Pal \\ Experimental Condensed Matter Physics Division, Saha Institute of Nuclear Physics, Bidhannagar, Kolkata 700064, India \\ Correspondence should be addressed to Barnana Pal, barnana.pal@saha.ac.in
}

Received 1 December 2011; Accepted 27 December 2011

Academic Editors: H. D. Hochheimer, L. Pusztai, and V. Stephanovich

Copyright ( 2012 Barnana Pal. This is an open access article distributed under the Creative Commons Attribution License, which permits unrestricted use, distribution, and reproduction in any medium, provided the original work is properly cited.

Cluster formation in a two-dimensional Lennard-Jones system under different conditions of temperature $(T)$ and particle concentration $(c)$ has been studied using the Monte-Carlo method with the introduction of real thermal motion of the constituent particles through a modification of the conventional Metropolis algorithm. The $c$ - $T$ phase diagram determined from the study of the root mean square displacement of the particles shows features characteristics of the $P-T$ diagram for phase equilibrium in real systems. The solid-like to liquid-like transition takes place when the average nearest neighbour distance increases by $\sim 1 \%$ of the equilibrium value in the low-temperature solid-like configuration. The Lindemann parameter $(\delta)$ is found to decrease with the increase of $c$ to reach a steady value of $\delta=0.0106 \pm 0.0004$ for $c \geq 0.6$.

\section{Introduction}

The relaxation dynamics in Lennard-Jones(L-J) systems have been studied using Monte-Carlo(MC) method by various workers [1-8]. The study of cluster formation, growth, and ordering under different physical conditions is important both from theoretical and experimental point of view $[1,3,8]$. In fact, the phase diagram of L-J system has received much importance in recent years [9-11], and lot of theoretical and computational methods have been employed for the determination of different physical parameters of the system [12-16]. One important aspect of such studies is the verification of Lindemann rule for the melting of solids [1719]. Parameters like triple point [9], melting temperature [18] estimated from these studies indicate that, though there are discrepancies, L-J system is extremely useful in predicting many physical properties of some real systems.

The dynamical process leading to an equilibrium configuration starting from nonequilibrium one depends on external parameters like temperature $(T)$ and pressure $(P)$ as well as on internal parameter-like interaction potential function $(V)$. In a recent work [20], it has been shown that in a two-dimensional (2-D) system of monoatomic L-J particles, an ordered equilibrium configuration is attained only if $T$ is less than the depth of the potential function, and the relaxation time is proportional to $(\sqrt{ } T)^{-1}$. In the present work, a more extensive study has been done on the dynamical behaviour of the equilibration process using modified Monte-Carlo process considering temperature-dependent atomic displacements [20]. The nature of cluster formation and ordering under different conditions of temperature $(T)$ and particle concentrations $(c)$ has been investigated considering root mean square atomic displacements. The method is described briefly in Section 2, results are discussed in Section 3, and Section 4 gives the conclusion.

\section{Simulation Method}

We consider a system of $N$ monoatomic particles confined in a 2-D square space with constant area LXL representing a configuration with concentration $c=N / L^{2}$. The particles interact with each other through L-J potential given by

$$
V(r)=\varepsilon\left[\left(\frac{r_{o}}{r}\right)^{12}-2\left(\frac{r_{o}}{r}\right)^{6}\right]
$$

where $\varepsilon$ measures the depth of the potential function, and $r_{o}$ gives the position of potential minimum. For the simulation process, we take $\varepsilon=1$ and $r_{o}=1$. The particles are allowed to take any position inside this area. To generate the initial configuration, the particles are placed at random positions in the 2-D space, and this represents a high 


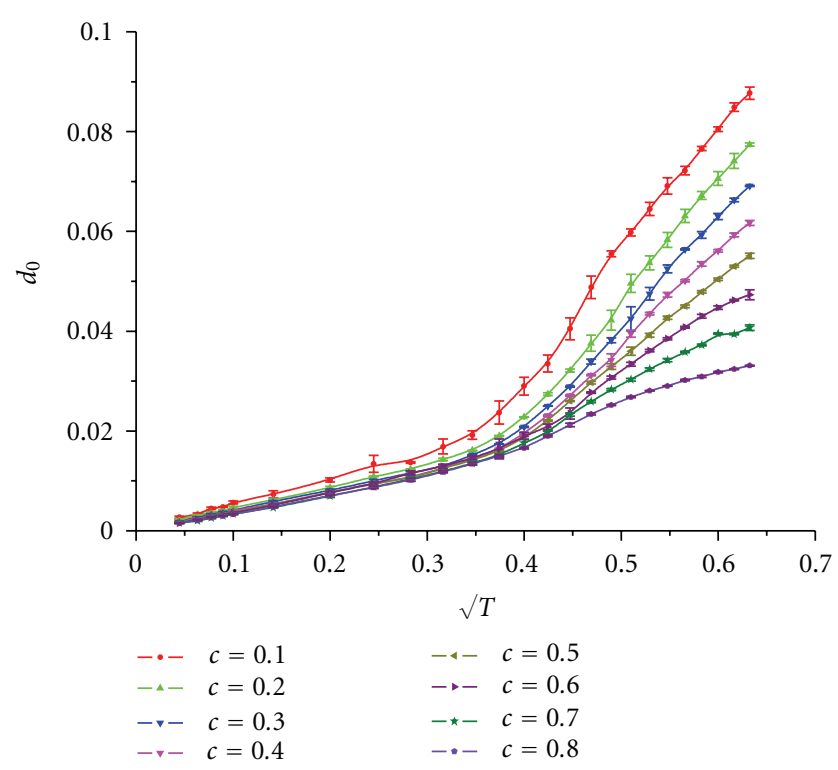

(a)

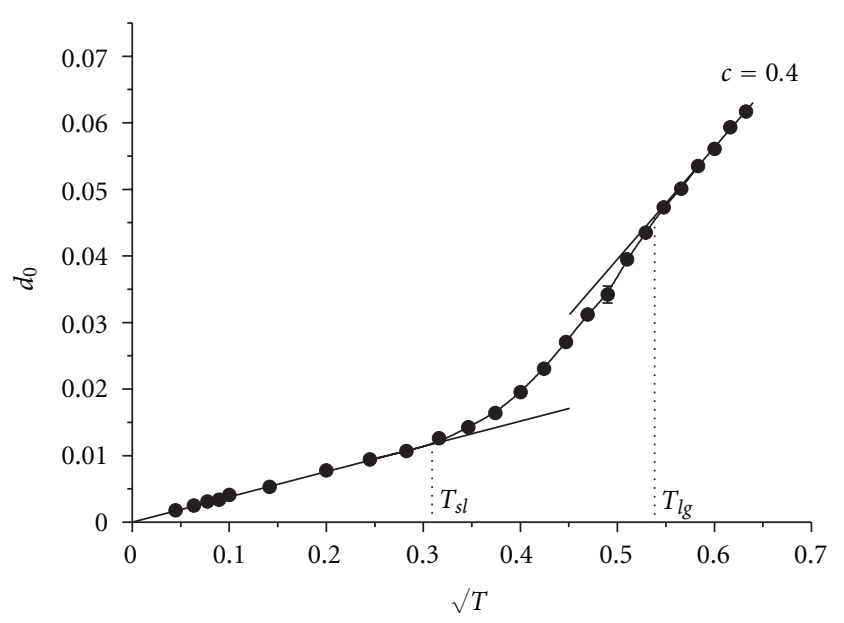

(b)

FIgURE 1: Average rms displacement $d_{o}$ as function of $\sqrt{ } T$. (a) presents the variation for $c=0.1,0.2,0.3,0.4,0.5,0.6,0.7$, and 0.8 and (b) presents the same for $c=0.4$ separately showing the transition temperatures $T_{\mathrm{sl}}$ and $T_{\mathrm{lg}}$.

temperature-disordered state. The system is then allowed to cool down suddenly. The simulation process representing the dynamical evolution of the system is generated by Metropolis algorithm, modified to introduce temperature-dependent movement of the constituent particles, as described in [20]. The root mean square deviation $(d)$ of the particles from their respective previous positions is determined as function of MC steps per particle $S$ for different values of $T$ and $c \cdot T$ is measured in units of $\varepsilon(T \equiv k T / \varepsilon)$. Assuming the evolution process to be exponential one, the average value of the rms displacement at equilibrium is determined using standard curve fitting method. For the present simulation, we take $L=14$ and generate configurations at eight different concentrations, $c=0.1,0.2,0.3,0.4,0.5,0.6,0.7$, and 0.8 . At each concentration minimum three configurations are generated to calculate the configuration average of the equilibrium rms displacement $d_{o}$. If fluctuation is more, average is taken over five configurations. The modified Metropolis algorithm [20] leads to quick equilibration, and 25000 iterations/particle at each temperature are found to be sufficient to equilibrate the system. At low $T$ and low $c$ clusters of different sizes are formed. The cluster size distribution has been determined considering a larger system size with $L=$ 30 , based on simulations on fifty different initial random particle configurations.

\section{Results and Discussion}

The configuration average value of the rms displacement $d_{o}$ plotted as function of $\sqrt{ } T$ for various particle concentrations is depicted in Figure 1(a). To have a clear view of the nature of variation, the plot for $c=0.4$ is shown separately in Figure 1(b). We observe that the variation of $d_{0}$ with $\sqrt{ } T$ is linear in the low- and high-temperature region. In the lowtemperature region, the system reaches a perfectly ordered configuration similar to crystalline solid. We designate this low-temperature phase as solid-like (SL). In the hightemperature region, the particles are more or less free to take any position inside the 2-D space, and no long- or shortrange order is observed in the system. We designate this phase as gas-like (GL). In between these two $d_{0}$ versus $\sqrt{ } T$ plot shows nonlinear behaviour. In this temperature region, local short-range order is observed, and this phase is designated as liquid-like (LL). The particle configurations in these phases are depicted in Figure 2. Figure 2(a) shows the initial disordered high-temperature configuration. Figures 2(b), 2(c), and 2(d) show the superimposed particle positions of 50 configurations taken at an interval of 50 iterations per particle at equilibrium at temperatures $T=0.04,0.16$, and 0.36 , respectively. We see that at $T=0.04$ the particles arrange themselves in a stable ordered solid-like structure. At $T=0.16$, particle movements are larger at equilibrium and local short-range order can be identified; the behaviour is liquid-like. At $T=0.36$, the particles are free to move throughout the space showing gas-like behaviour.

The temperatures, at which the $d_{0}$ versus $\sqrt{ } T$ curve starts deviating from linear behaviour, are considered as the transition temperatures. SL to LL transition temperature $T_{\mathrm{sl}}$ and LL to GL transition temperature $T_{\mathrm{lg}}$ are depicted in Figure 1(b). The transition temperature as function of concentration $(c)$ is shown in Figure 3. The bars represent the estimated error in finding the transition temperatures. $\mathrm{AB}$ represents the SL-LL transition line, and MN represents the LL-GL transition line. We see that $T_{\mathrm{sl}}$ remain almost same for all the concentration values. On the other hand, $T_{\lg }$ increases with the increase of $c$ and attains a constant value for $c \geq 0.6$. 


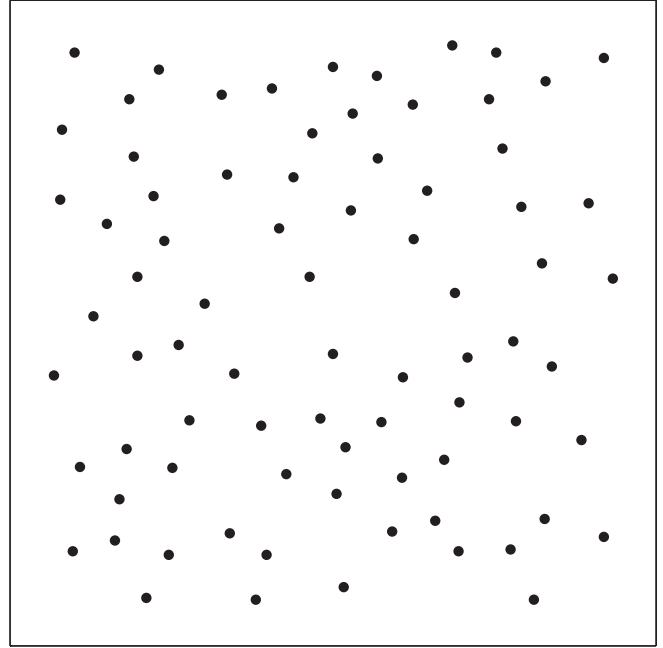

(a) Initial configuration

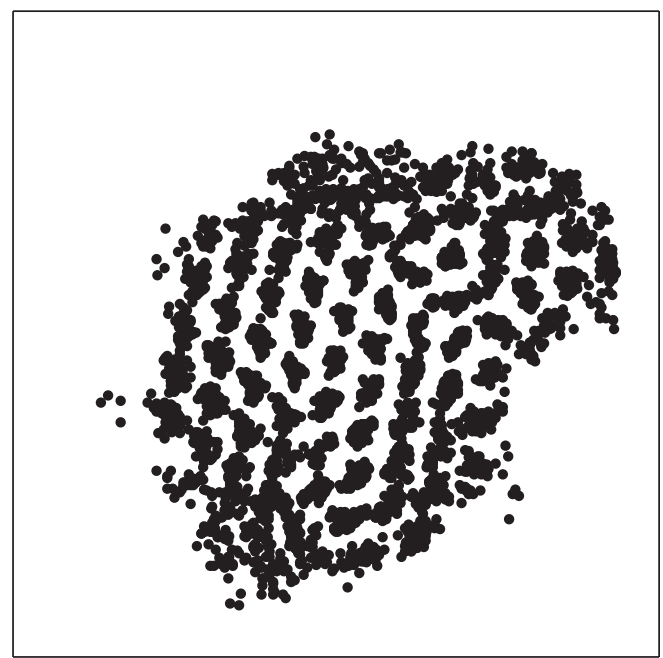

(c) $T=0.16$

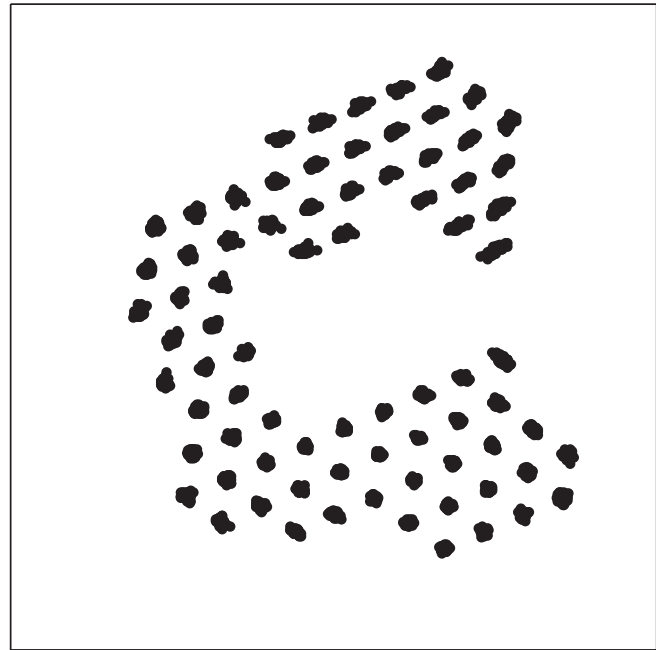

(b) $T=0.04$

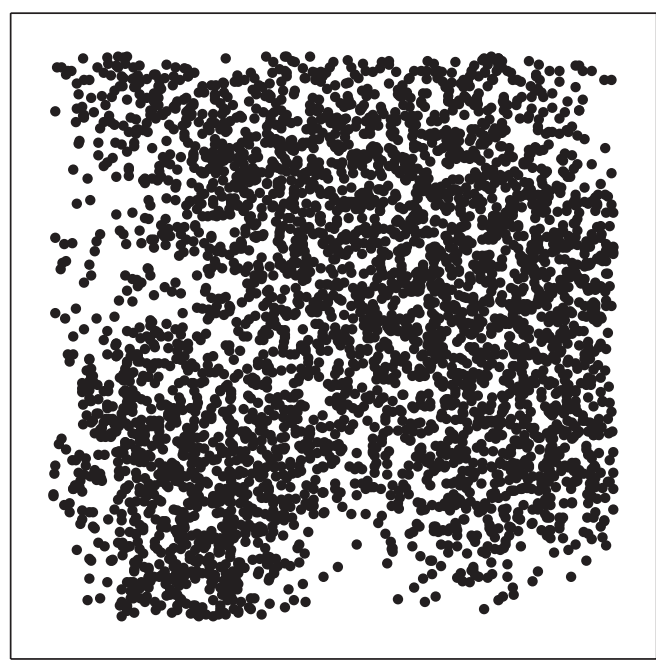

(d) $T=0.36$

Figure 2: Particle configurations at different temperatures. Initial random configuration representing high-temperature disordered state is depicted by (a). The superimposed particle positions of 50 configurations at equilibrium taken at an interval of 50 iterations per particle at temperatures $T=0.04,0.16$, and 0.36 are presented in (b), (c), and (d), respectively.

In fact identification of $T_{\text {lg }}$ becomes difficult for higher $c$ values. This is a consequence of the fact that the particles are confined in a space with constant area, and at relatively high $T$ this represents a situation where the system pressure becomes very high. The particles do not have much space for free movement, and there is possibility that the system remains in LL phase. Extrapolation of $\mathrm{AB}$ and $\mathrm{MN}$ meet at a point $\mathrm{P}$ (the triple point) with $T=0.08$ and $c=0.02$. It is interesting to note the similarity of the $c-T$ diagram with the $P-T$ diagram for phase equilibrium [21]. The highertemperature region of $\mathrm{MN}$ for $c \geq 0.6$, where $T_{\mathrm{lg}}$ does not change with $c$, may be indicative for the existence of the critical point with $T_{c}=0.3$.

Figure 4 presents the temperature variation of average nearest neighbour distance $\left(d_{\text {nn }}\right)$ calculated over ten configurations at equilibrium near SL-LL transition (indicated by dotted line) for $c=0.4$ and 0.8 . The $d_{\mathrm{nn}}$ versus $\sqrt{ } T$ plot shows a distinct change in the slope at the SL-LL transition point where $d_{\mathrm{nn}}$ is found to have a value larger by only $\sim 1 \%$ from its equilibrium value in the low-temperature ordered SL phase. According to Lindemann rule, solid-to-liquid transition takes place when the Lindemann parameter $\delta$, defined as the ratio of rms fluctuation in atomic positions about the equilibrium lattice positions and the nearest neighbour lattice distance exceeds a threshold value $[17-19,22]$. The value of $\delta$ is not constant but depends on factors like crystal structure, nature of interaction potential and other quantum effects $[23,24]$. The reported values of $\delta$ vary from 0.05 to 0.2 [22]. In the present study, we have calculated $\delta$ for different concentrations, and the result is depicted in Figure 5. We observe that $\delta$ decreases with the increase of $c$ and attains a constant value $\delta=0.0106 \pm 0.0004$ for $c \geq 0.6$. The value of $\delta$ is lower than those reported in the literature. This may be caused by the lower dimensionality of the system. 


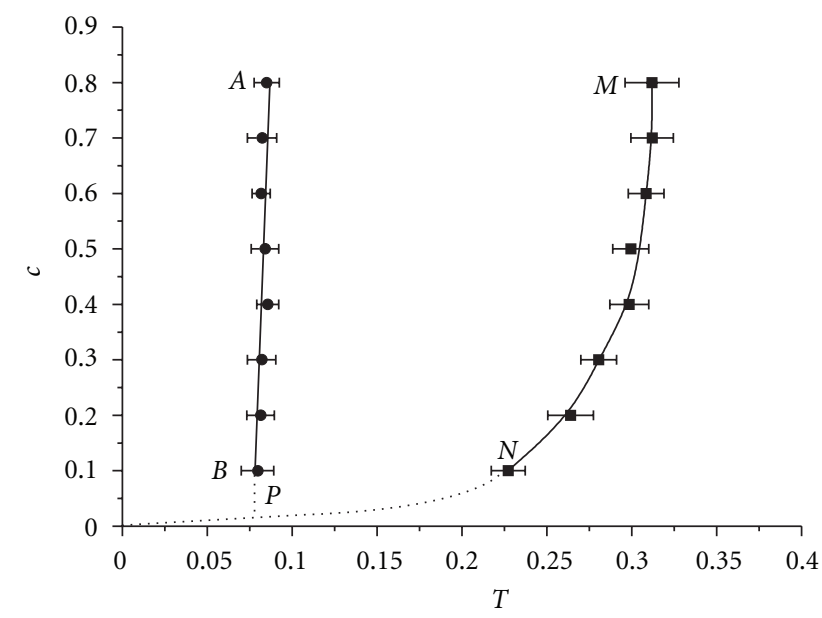

Figure 3: Concentration $(c)$ versus temperature $(T)$ phase diagram. AB represents SL-LL transition temperatures $T_{\mathrm{sl}}$, and MN represents LL-GL transition temperatures $T_{\mathrm{lg}}$. On extrapolation $\mathrm{AB}$ and MN meet at $P$, where $T \cong 0.8$ and $c \cong 0.02$.

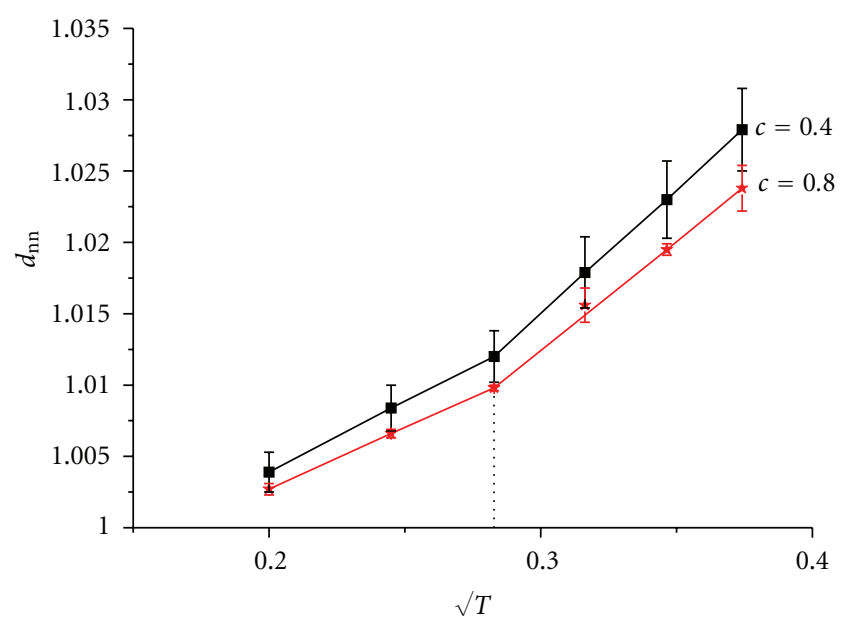

FIgURE 4: Average nearest neighbour distance $\left(d_{\mathrm{nn}}\right)$ as function of $\sqrt{ } T$ for $c=0.4$ and 0.8 . Dotted line indicates the position of SL-LL transition.

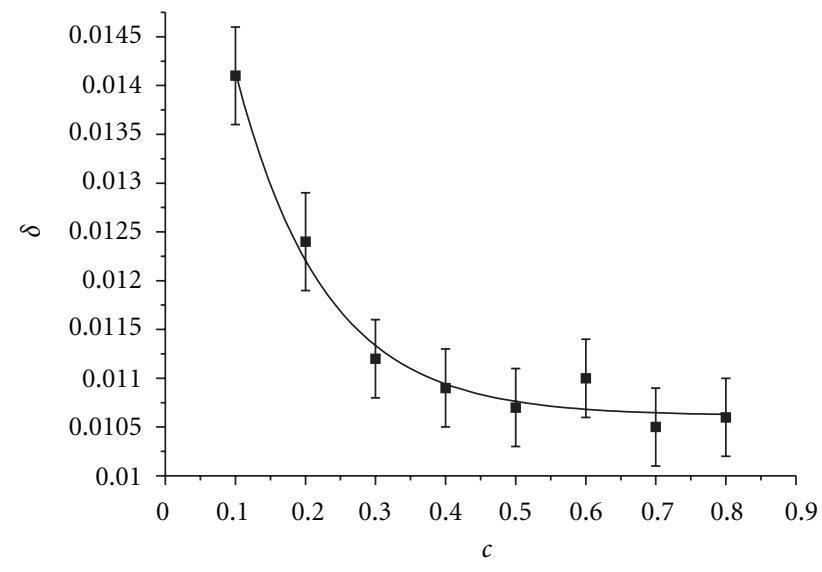

Figure 5: Lindemann parameter $\delta$ as function of concentration $c$. 

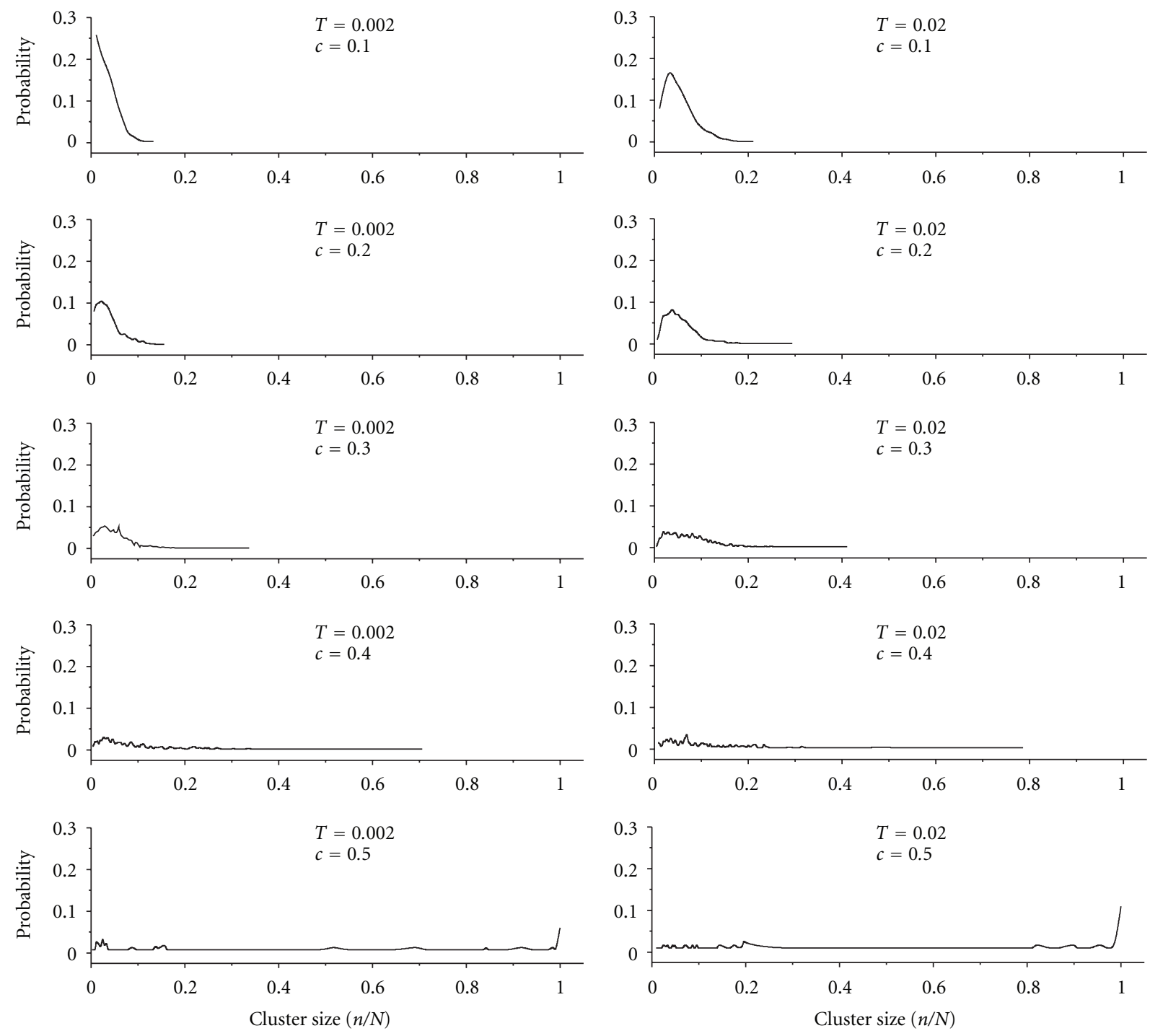

(a)
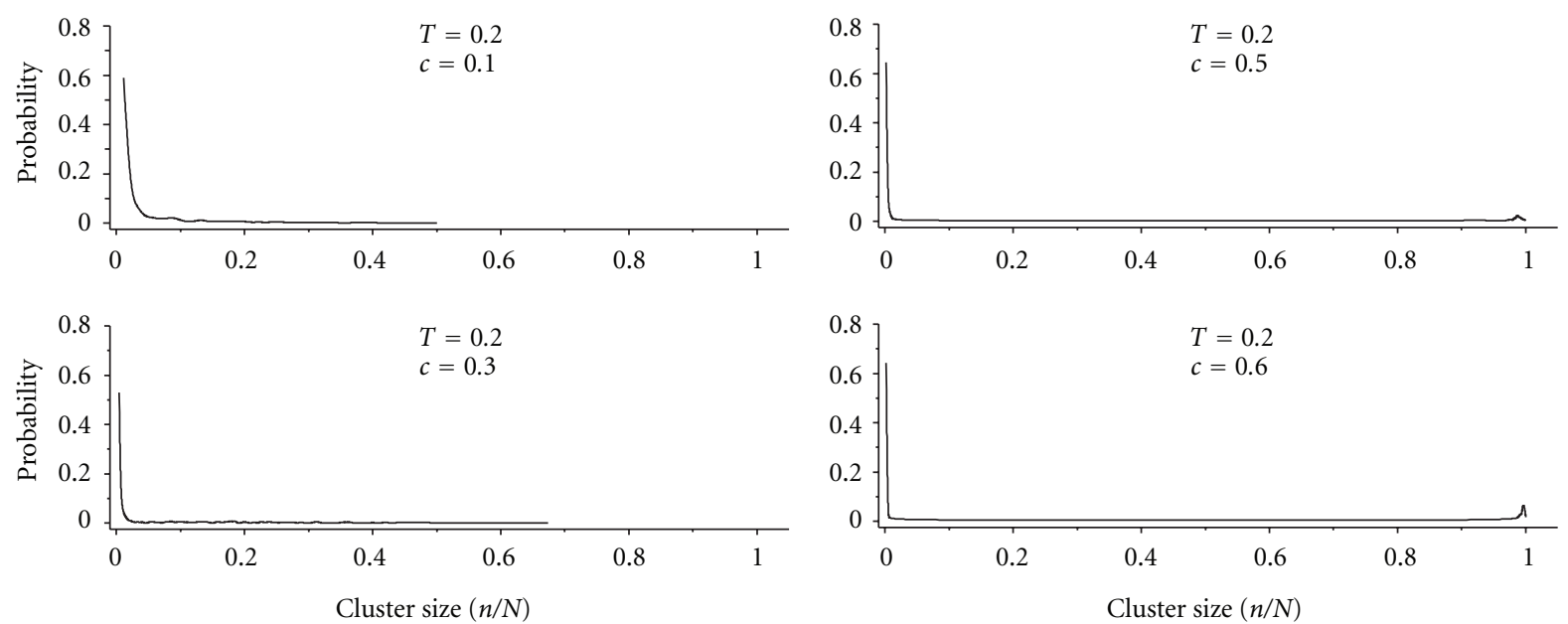

(b)

Figure 6: Probability distribution function for (a) concentrations $c=0.1,0.2,0.3,0.4$, and 0.5 at $T=0.002$ and 0.02 and for (b) $c=$ $0.1,0.3,0.5 \& 0.6$ at $T=0.2$. 
The system of particles is allowed to cool down suddenly from a high-temperature disordered state to a lower temperature. We observe that at low-concentration clusters of various sizes are formed in a scattered way. At very lowtemperature particle movements are small and only those finding another one in the range of L-J interaction potential join together to form clusters. The distributions of cluster size under different particle concentrations are determined at temperatures $T=0.002,0.02$, and 0.2 . For this, we generate 50 different initial configurations in a two-dimensional space with $L=30$ and find out the probability of getting clusters of different sizes at equilibrium. A cluster is defined as an assembly of particles in which each particle has at least one neighbour within a distance $1.5 r_{o}$. At this distance the potential function has a value less than $20 \%$ of the peak value, and the particle can be considered more or less free to move. For normalization, cluster of $n$ particles is assumed to be of size $n / N$. The probability distribution functions are presented in Figure 6. In Figure 6(a) we have shown the distribution function for $c=0.1,0.2,0.3,0.4$, and 0.5 for $T=0.002$ and 0.02 . We see that, at very low temperature, $T=0.002$, the probability of forming cluster of smaller size is larger at low concentration, $c=0.1$. For $c=0.2$ a peak is observed in the distribution function. With the increase of $c$, the height of the peak decreases and the probability of finding larger cluster increases. For $c=0.5$ there is finite probability of getting a single cluster $($ size $=1)$. For $c>0.5$ the particles form a single cluster. For higher temperature, $T=0.02$, a peak is observed in the distribution function even at low concentration, $c=$ 0.1 . Also the probability of formation of a single cluster at $c=0.5$ is larger. This is expected since in our simulation process we have introduced temperature-dependent particle movement and at larger $T$ the particle movements are larger so that the probability of finding another particle in the interaction range of L-J potential to form cluster is greater. There is a critical concentration lying between 0.5 and 0.6 above which all particles join to form a single cluster. In Figure 6(b) probability distribution for $c=0.1,0.3,0.5$, and 0.6 at $T=0.2$ are presented. This temperature is relatively high, and there is always a finite probability of formation of small size clusters. As a result a small peak is observed for $n / N$ a little lower than 1 at higher concentrations $c=0.5$ and 0.6.

One important point to be noted is that, the present simulation leads to SL structures at low temperature $T<T_{\mathrm{sl}}$, and long-range order is present in this phase which is clearly depicted in Figure 2(b). This is in contradiction to Mermin's theory [25] showing that crystalline long-range order in 2dimensions is impossible for L-J type power-law potentials. However, for hard-core type potentials, Mermin's theory is inconclusive. The observed discrepancy may be due to the fact that in the simulation process the L-J potential effectively behaves like hard-core potential due to strong repulsion for $r$ sufficiently less than $r_{o}$.

\section{Conclusion}

We have investigated the clustering and ordering in 2dimensional L-J system under different conditions of temperature and particle concentration using a modified
Monte-Carlo method introducing temperature-dependent particle movements. The phase diagram determined from a study of the temperature dependence of the rms distance of the constituent particles show similarity with the $P-T$ diagram for phase equilibrium. An estimate of the triple point coming out of this study is $T=0.08$ and $c=0.02$ and the critical temperature $T_{c}=0.3$. The Lindemann parameter $\delta$ calculated at the SL to $\mathrm{LL}$ transition temperature is found to be concentration dependent. With the increase of concentration, $\delta$ decreases to reach a constant value $\delta=0.0106 \pm 0.0004$ for $c \geq 0.6$. The $\delta$ value is lower than those reported in the literature. This may be caused by the lower dimensionality (2-D) of the system concerned.

Regarding cluster size distribution, it is observed that, at low temperature and low concentration, probability of formation of smaller clusters is larger. With a little increase in the particle concentration, the probability of formation of clusters of an optimum size is maximum. A further increase in the concentration increases the probability of finding clusters of larger size resulting in a decrease in the probability of finding clusters of optimum size. At somewhat higher temperature, the probability of finding clusters of optimum size is maximum even at very low concentration. At low and moderate temperature, there is a critical concentration between 0.5 and 0.6 , above which all the constituent particles join together to form a single cluster. With further increase in the temperature, there is always a finite probability for the formation of smaller size clusters even at relatively high particle concentration. This is quite natural in liquid-like phase.

According to Mermin's theory crystalline order in 2 dimensions is impossible for L-J type potentials. The present simulation, however, leads to ordered structure at low temperature. The observed discrepancy may be caused by the fact that due to strong repulsion for $r$ sufficiently less than $r_{o}$, L-J potential behaves effectively like hard-core potential for which Mermin's theory leads to inconclusive result.

\section{References}

[1] N. G. Garcia and J. M. S. Torroja, "Monte Carlo calculation of argon clusters in homogeneous nucleation," Physical Review Letters, vol. 47, no. 3, pp. 186-190, 1981.

[2] S. M. Kathmann and B. N. Hale, "Monte Carlo simulations of small sulfuric acid-water clusters," Journal of Physical Chemistry B, vol. 105, no. 47, pp. 11719-11728, 2001.

[3] Y. Kataoka and Y. Yamada, "Monte Carlo simulation on the free energy of homogeneous nucleation in the supersaturated Lennard-Jones vapor phase," Fluid Phase Equilibria, vol. 194197, pp. 207-217, 2002.

[4] B. N. Hale and D. J. DiMattio, "Scaling of the nucleation rate and a Monte Carlo discrete sum approach to water cluster free energies of formation," Journal of Physical Chemistry B, vol. 108, no. 51, pp. 19780-19785, 2004.

[5] A. Lauri, J. Merikanto, E. Zapadinsky, and H. Vehkamäki, "Comparison of Monte Carlo simulation methods for the calculation of the nucleation barrier of argon," Atmospheric Research, vol. 82, no. 3-4, pp. 489-502, 2006.

[6] J. Merikanto, E. Zapadinsky, A. Lauri, and H. Vehkamäki, "Origin of the failure of classical nucleation theory: incorrect 
description of the smallest clusters," Physical Review Letters, vol. 98, no. 14, Article ID 145702, 2007.

[7] M. Schrader, P. Virnau, and K. Binder, "Simulation of vaporliquid coexistence in finite volumes: a method to compute the surface free energy of droplets," Physical Review E, vol. 79, no. 6, Article ID 061104, 2009.

[8] B. N. Hale and M. Thomason, "Scaled vapor-to-liquid nucleation in a lennard-jones system," Physical Review Letters, vol. 105, no. 4, Article ID 046101, 2010.

[9] S. A. Khrapak, M. Chaudhuri, and G. E. Morfill, "Liquid-solid phase transition in the Lennard-Jones system," Physical Review $B$, vol. 82, no. 5, Article ID 052101, 2010.

[10] E. A. Mastny and J. J. de Pablo, "Melting line of the LennardJones system, infinite size, and full potential," Journal of Chemical Physics, vol. 127, no. 10, Article ID 104504, 2007.

[11] H. Okumura and F. Yonezawa, "Liquid-vapor coexistence curves of several interatomic model potentials," Journal of Chemical Physics, vol. 113, no. 20, pp. 9162-9168, 2000.

[12] L. Verlet, "Computer "experiments" on classical fluids. I. Thermodynamical properties of Lennard-Jones molecules," Physical Review, vol. 159, no. 1, pp. 98-103, 1967.

[13] J. A. Barker and D. Henderson, "Perturbation theory and equation of state for fluids. II. A successful theory of liquids," The Journal of Chemical Physics, vol. 47, no. 11, pp. 4714-4721, 1967.

[14] J. P. Hansen, "Phase transition of the Lennard-Jones system. II. High-temperature limit," Physical Review A, vol. 2, no. 1, pp. 221-230, 1970.

[15] J. K. Johnson, J. A. Zollweg, and K. E. Gubbins, “The LennardJones equation of state revisited," Molecular Physics, vol. 78, pp. 591-618, 1993.

[16] R. Agrawala and D. A. Kofkea, "Thermodynamic and structural properties of model systems at solid-fluid coexistence II. Melting and sublimation of the Lennard-Jones system," Molecular Physics, vol. 85, p. 43, 1995.

[17] F. A. Lindemann, "The calculation of molecular vibration frequencies," Zeitschrift für Physik, vol. 11, pp. 609-912, 1910.

[18] A. C. Lawson, D. P. Butt, J. W. Richardson, and J. Li, “Thermal expansion and atomic vibrations of zirconium carbide to 1600 K,” Philosophical Magazine, vol. 87, no. 17, pp. 2507-2519, 2007.

[19] M. Ross, "Generalized Lindemann melting law," Physical Review, vol. 184, no. 1, pp. 233-242, 1969.

[20] B. Pal, "Relaxation dynamics in small clusters: a modified Monte Carlo approach," Journal of Computational Physics, vol. 227, no. 4, pp. 2666-2673, 2008.

[21] M. N. Saha and B. N. Srivastava, A Treatise on Heat, Indian Press, Allahabad, India, 1958.

[22] C. Chakravarty, P. G. Debenedetti, and F. H. Stillinger, "Lindemann measures for the solid-liquid phase transition," Journal of Chemical Physics, vol. 126, no. 20, Article ID 204508, 2007.

[23] S. N. Luo, A. Strachan, and D. C. Swift, "Vibrational density of states and Lindemann melting law," Journal of Chemical Physics, vol. 122, no. 19, Article ID 194709, pp. 1-5, 2005.

[24] F. H. Stillinger and T. A. Weber, "Lindemann melting criterion and the Gaussian core model," Physical Review B, vol. 22, no. 8, pp. 3790-3794, 1980.

[25] N. D. Mermin, "Crystalline order in two dimensions," Physical Review, vol. 176, no. 1, pp. 250-254, 1968. 

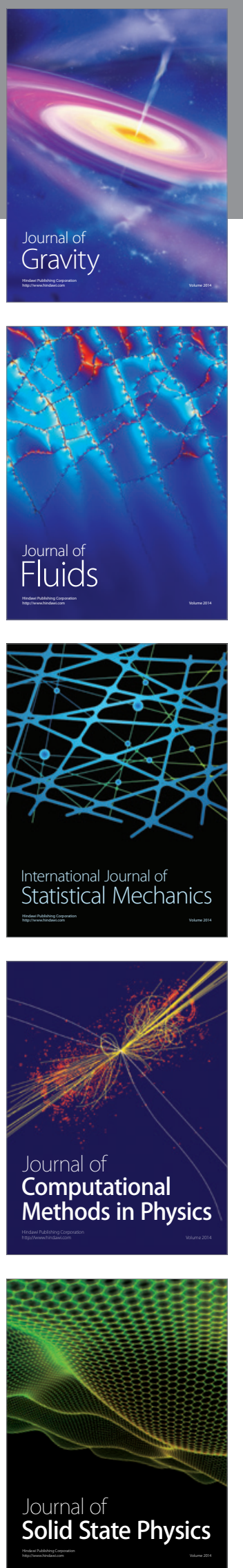
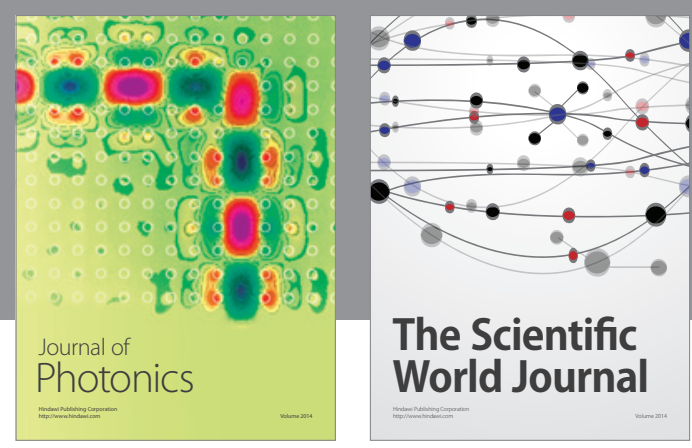

The Scientific World Journal

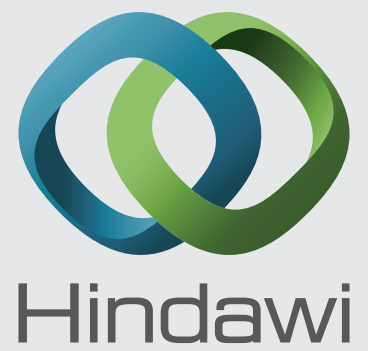

Submit your manuscripts at http://www.hindawi.com
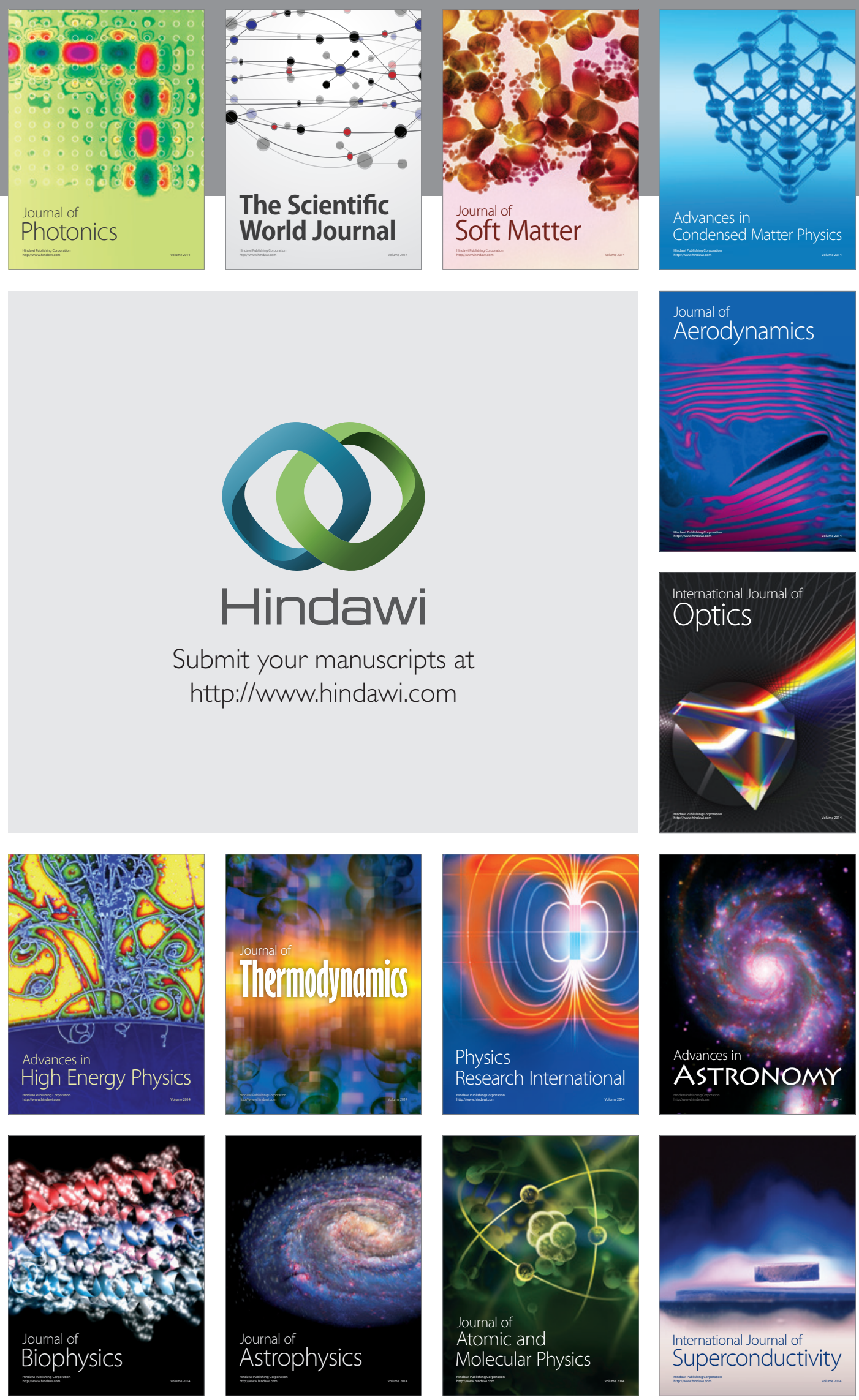
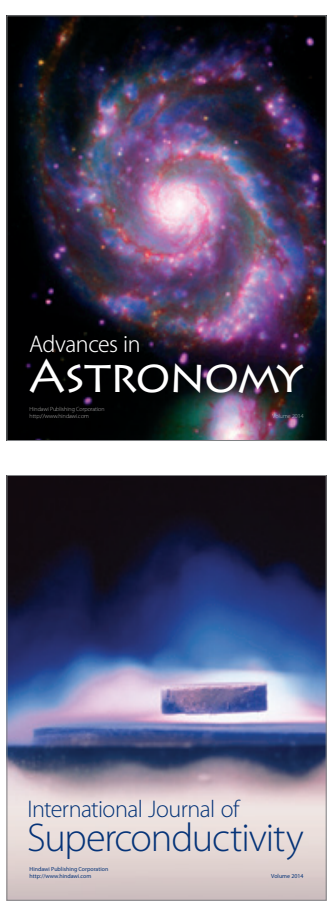